\title{
GabiPD: The GABI Primary Database - a plant integrative 'omics' database
}

Diego Mauricio Riaño-Pachón ${ }^{1,2}$, Axel Nagel ${ }^{1}$, Jost Neigenfind ${ }^{1}$, Robert Wagner ${ }^{1}$, Rico Basekow $^{1}$, Elke Weber $^{1}$, Bernd Mueller-Roeber ${ }^{2}$, Svenja Diehl ${ }^{3}$, Birgit Kersten ${ }^{1,2, \S}$

${ }^{1}$ GabiPD team, Bioinformatics group, Max Planck Institute of Molecular Plant Physiology, Am Mühlenberg 1, 14476 Potsdam-Golm, Germany.

${ }^{2}$ Department of Molecular Biology, University of Potsdam, Karl-Liebknecht-Str. 24-25, Haus 20, 14476 Potsdam-Golm, Germany.

${ }^{3}$ Former RZPD German Resource Center for Genome Research GmbH, Berlin, Germany.

${ }^{\S}$ Corresponding author, kersten@mpimp-golm.mpg.de; Max Planck Institute of Molecular Plant Physiology, Am Mühlenberg 1, 14476 Potsdam-Golm, Germany 


\section{ABSTRACT}

The GABI Primary Database, GabiPD (http://www.gabipd.org/), was established in the frame of the German initiative for Genome Analysis of the Plant Biological System (GABI). The goal of GabiPD is to collect, integrate, analyse and visualise primary information from GABI projects. GabiPD constitutes a repository and analysis platform for a wide array of heterogeneous data from high-throughput experiments in several plant species. Data from different 'omics' fronts are incorporated (i.e., genomics, transcriptomics, proteomics and metabolomics), originating from 14 different model or crop species. We have developed the concept of GreenCards for text based retrieval of all data types in GabiPD (e.g., clones, genes, mutant lines). All data types point to a central Gene GreenCard, where gene information is integrated from genome projects or NCBI UniGene sets. The centralised Gene GreenCard allows visualising ESTs aligned to annotated transcripts as well as displaying identified protein domains and gene structure. Moreover GabiPD makes available interactive genetic maps from potato and barley, and 2DE-gels from Arabidopsis thaliana and Brassica napus. Gene expression and metabolic profiling data can be visualised through MapManWeb. By the integration of complex data in a framework of existing knowledge, GabiPD provides new insights and allows for new interpretations of the data. 


\section{INTRODUCTION}

Experimental studies in the post genomic era generate a very large amount of data from highthroughput experiments on biological systems. Current studies include, among others, expression and metabolite profiles, proteome and interaction data (e.g., DNA-protein and protein-protein interactions), collected at different space and time scales. This increasing flow of data requires computational systems that, beside managing efficiently the enormous quantity of data, are capable of integrating and displaying these disparate data collections in a meaningful and amicable way. We have developed the GABI Primary Database, GabiPD, in order to fulfil these requirements.

GabiPD is a web accessible database that was developed in the frame of the German initiative for Genome Analysis of the Plant Biological System (Genomanalyse im biologischen System Pflanze, GABI). GabiPD allows a seamless integration of varied 'omics' data types obtained from plant systems and follows the MIAME (1) and MIAMET (2) standards for storing gene expression and metabolic profiling data, respectively. Its flexible design allows for a high level of data integration, and eases cross-referencing the different GabiPD data types among each other (e.g., mapping information, sequences and SNP data, 2DE-gel images and protein information) and to public gene/protein-specific information which in turn provides the users a comprehensive overview of the available information for their particular gene or protein of interest. The integration with genome databases like TAIR (3) and general nucleotide databases like GenBank, as well as cross-links to secondary databases, such as ARAMEMNON (4), PlnTFDB (5), GABI-KAT (6), PhosPhAt (7), and ProMEX (8) further increase the usefulness of GabiPD.

\section{METHODS AND CONTENTS}

\section{DESIGN AND IMPLEMENTATION}

GabiPD's web interface was developed using Perl and Java in combination with template processing to separate the visualisation from the application logic. Our applications are database driven, which means that the application interface logic is derived directly from the database structure (shown in blue in Figure 1). To achieve this, we deploy reverse engineering methods in combination with template processing to generate interfaces to programming languages like Perl or Java, thus supporting all the database-specific actions like 'insert', 'update', 'delete' or 'select'. These object-oriented interfaces are automatically generated from the database schema, supporting inheritance, automated key generation and advanced exception handling. The separation of database application interface and application logic (shown in yellow in Figure 1) facilitates fast adjustment to modifications of the data structure and diminishes the efforts on fixing existing application logic during larger database changes.

\section{GabiPD CONTENT AND GENE-CENTRIC VIEWS}

Currently GabiPD includes data originating from 14 different angiosperm species representing the most important lineages in the flowering plants (see Figure 2). Arabidopsis thaliana is the most widely represented model species, followed by the crop plants Solanum tuberosum (potato) and Hordeum vulgare (barley). In GabiPD, genomic, transcriptomic, proteomic and metabolomic data are integrated from those species. Genomic data comprise mapping information, sequences and SNP/InDel information. Transcriptomics is represented by a large number of ESTs and corresponding sequence trace files. ESTs are further analysed by BLAST and ORF analysis. For barley, in addition, EST clustering results and corresponding information on a new $27 \mathrm{~K}$ unigene set are accessible and downloadable. As a 
type of proteomic data, annotated 2DE-gel images from Arabidopsis thaliana and Brassica napus are integrated. Moreover, transcript and metabolite profiling data are provided via MapManWeb.

Most entries of all GabiPD data types are pointing to the central Gene GreenCard and vice versa (see Figure 3 and next section). In the Gene GreenCard, gene information from genome annotation projects or NCBI UniGene sets is integrated and useful links to secondary databases are provided. Currently the genome annotation (TAIR version 7.0) for A. thaliana (3) is integrated. Annotations for other sequenced species will follow. In order to ease the transfer of knowledge from sequenced to non-sequenced species, i.e., crop plants, we have performed similarity-based mappings between closely related species, i.e., Arabidopsis and Brassica spp.

\section{QUERYING THE DATABASE}

We have developed the concept of GreenCards as a central entrance for text-based data queries and visualisation which grant public as well as credentials-based access to the integrated data in GabiPD. GreenCards enable users to comprehensively query GabiPD by genotype name, marker or gene name, keyword or GenBank sequence accession number. Searches can be restricted to selected species or data types, while wildcards can be used to broaden the scope of the query. The result of a GreenCard search is presented as a list of hits with links to complete descriptions, i.e., GreenCards. Figure 3 shows an example of this type of search, where the user had entered the gene name 'FLOWERING LOCUS T' as a search term. This search retrieves, among others, an Arabidopsis Gene GreenCard (gene: AT1G65480.1) corresponding to the genome annotation project and Plant GreenCards representing mutant plant lines, e.g., plant 290E08, that has BLAST hits to the gene AT1G65480.1 and with seeds available from GABI-KAT (6). Moreover, several Clone GreenCards of cDNA clones (e.g., clone: MPMGp2011E01215) in which the keyword is found were retrieved by the search. A more strict relationship between the Gene GreenCard and the Plant and Clone GreenCards is established by similarity-based searches. The best BLAST hit of the sequence, e.g., relationship to a cDNA clone or a mutant plant line, appears in the section Related with, and the users can go from the clone or the plant line to the associated gene or vice versa.

Furthermore, the Gene GreenCard, which displays information from genome annotation projects and NCBI UniGene sets, has been extended to include links to secondary databases, such as ARAMEMNON (4), GABI-KAT (6), and ProMEX (8). Additionally, schematic representations of gene sequence features are provided to highlight protein domains identified using the latest PFAM library (9), exon-exon borders and untranslated regions (UTRs) identified by the genome annotation projects (Figure 3). These features are displayed onto a representation of the cDNA sequence.

Alternatively, users can enter their own amino acid or nucleotide sequence to identify by a BLAST search (10) similar sequences integrated in GabiPD.

In addition to the GreenCard and BLAST search functionality, users can browse and search the genetic maps and 2DE-gels stored in GabiPD, through specifically designed visualisation tools: (i) 2DEGelViewer by which 2DE-gel images can be viewed in an interactive way, which allows retrieving extra information on 2D-spots as identified by mass spectrometry (Figure 3); (ii) genetic mapping data can be visualised using YAMB (Yet Another Map Browser; Figure 4) with the possibility to view details on all mapped elements (11); (iii) MapManWeb (Figure 5), allows the visualisation and extraction of relevant information from transcript and metabolite profiling data and the graphical mapping of such data onto diagrams of metabolic pathways and other biological processes (12); and (iv) an extended version of JTrev (13) allows the display of sequence traces with integrated SNP information. 
The GabiPD project page serves as an additional gateway to specific data by providing project specific views, such as BreedCAM or PoMaMo (11), where potato genomic data and Solanaceae function maps for pathogen resistance are accessible.

\section{ADDITIONAL TOOLS AVAILABLE FROM GabiPD}

In addition to the data and data visualisation available from our site, the newest versions of the following tools are made available for download:

MapMan - desktop version: a user-driven software tool that displays large datasets (e.g., gene expression data from Arabidopsis Affymetrix arrays) onto diagrams of biological processes, such as metabolic pathways (12).

SATlotyper: a software tool designed for inferring haplotypes and phased genotypes from unphased SNP data for polyploid and polyallelic heterozygous populations (14).

\section{FUTURE DIRECTIONS}

The presentation of a wide spectrum of different plant species in GabiPD paves the way for cross-species comparisons that are facilitated by the availability of BLAST hits between the GabiPD sequences and plant NCBI UniGene sets. To ease the transfer of knowledge from sequenced to non-sequenced plant species, the genome annotations of Oryza sativa, Populus trichocarpa and Vitis vinifera will be added and mapped to closely related species in the near future. By that useful information about orthologous genes will be included for cross-species studies. Moreover, we will extend our WebServices to provide programmatic access to multiple data types for all plant species in GabiPD.

\section{FUNDING}

This work was supported by the German Ministry for Education and Research (BMBF) [GABI I: 0312272, GABI II: 0313112 and GABI-FUTURE: 0315046], the former German Resource Center for Genome Research (RZPD) GmbH and the Max Planck Society.

\section{ACKNOWLEDGEMENTS}

The GabiPD team thanks the GABI and the WPG (Wirtschaftsverbund Pflanzengenomforschung GABI e.V.) community for providing data and supporting the continuous development of the database. Dr. Björn Usadel is acknowledged for helpful discussions and his support in the development of MapManWeb. We wish to thank Dr. Patrick Schweitzer, Dr. Lothar Altschmied, Dr. Uwe Scholz and Dr. Nils Stein for the collaboration in the generation of the new $27 \mathrm{~K}$ barley unigene set, and Dr. Kathryn F. Beal for sharing the source code of the trace viewer JTrev. Özgür Demir and Sebastian Köhler are acknowledged for further developments of user interfaces and for data integration.

\section{REFERENCES}

1. Ball, C.A. and Brazma, A. (2006) MGED standards: work in progress. Omics, 10, 138-144.

2. Jenkins, H., Hardy, N., Beckmann, M., Draper, J., Smith, A.R., Taylor, J., Fiehn, O., Goodacre, R., Bino, R.J., Hall, R. et al. (2004) A proposed framework for the description of plant metabolomics experiments and their results. Nat Biotechnol, 22, 1601-1606. 
3. Swarbreck, D., Wilks, C., Lamesch, P., Berardini, T.Z., Garcia-Hernandez, M., Foerster, H., Li, D., Meyer, T., Muller, R., Ploetz, L. et al. (2008) The Arabidopsis Information Resource (TAIR): gene structure and function annotation. Nucleic Acids Res, 36, D1009-D1014.

4. Schwacke, R., Schneider, A., van der Graaff, E., Fischer, K., Catoni, E., Desimone, M., Frommer, W.B., Flugge, U.I. and Kunze, R. (2003) ARAMEMNON, a novel database for Arabidopsis integral membrane proteins. Plant Physiol, 131, 16-26.

5. Riaño-Pachón, D.M., Ruzicic, S., Dreyer, I. and Mueller-Roeber, B. (2007) PlnTFDB: An integrative plant transcription factor database. BMC Bioinformatics, 8, 42.

6. Li, Y., Rosso, M.G., Viehoever, P. and Weisshaar, B. (2007) GABI-Kat SimpleSearch: an Arabidopsis thaliana T-DNA mutant database with detailed information for confirmed insertions. Nucleic Acids Res, 35, D874-878.

7. Heazlewood, J.L., Durek, P., Hummel, J., Selbig, J., Weckwerth, W., Walther, D. and Schulze, W.X. (2008) PhosPhAt: a database of phosphorylation sites in Arabidopsis thaliana and a plant-specific phosphorylation site predictor. Nucleic Acids Res, 36, D1015-1021.

8. Hummel, J., Niemann, M., Wienkoop, S., Schulze, W., Steinhauser, D., Selbig, J., Walther, D. and Weckwerth, W. (2007) ProMEX: a mass spectral reference database for proteins and protein phosphorylation sites. BMC Bioinformatics, 8, 216.

9. Finn, R.D., Tate, J., Mistry, J., Coggill, P.C., Sammut, S.J., Hotz, H.R., Ceric, G., Forslund, K., Eddy, S.R., Sonnhammer, E.L. et al. (2008) The Pfam protein families database. Nucleic Acids Res, 36, D281-288.

10. Altschul, S.F., Gish, W., Miller, W., Myers, E.W. and Lipman, D.J. (1990) Basic local alignment search tool. J Mol Biol, 215, 403-410.

11. Meyer, S., Nagel, A. and Gebhardt, C. (2005) PoMaMo-a comprehensive database for potato genome data. Nucleic Acids Res, 33, D666-670.

12. Usadel, B., Nagel, A., Thimm, O., Redestig, H., Blaesing, O.E., Palacios-Rojas, N., Selbig, J., Hannemann, J., Piques, M.C., Steinhauser, D. et al. (2005) Extension of the visualization tool MapMan to allow statistical analysis of arrays, display of corresponding genes, and comparison with known responses. Plant Physiol, 138, 1195-1204.

13. Bonfield, J.K., Beal, K.F., Betts, M.J. and Staden, R. (2002) Trev: a DNA trace editor and viewer. Bioinformatics, 18, 194-195.

14. Neigenfind, J., Gyetvai, G., Basekow, R., Diehl, S., Achenbach, U., Gebhardt, C., Selbig, J. and Kersten, B. (2008) Haplotype inference from unphased SNP data in heterozygous polyploids based on SAT. BMC Genomics, 9, 356.

15. Soltis, P.S. and Soltis, D.E. (2004) The origin and diversification of angiosperms. Am J Bot, 91, 1614-1626.

16. Angiosperm Phylogeny Group. (2003) An update of the Angiosperm Phylogeny Group classification for the orders and families of flowering plants: APG II. Bot $J$ Linn Soc, 141, 399-436.

17. Knapp, S. (2002) Tobacco to tomatoes: a phylogenetic perspective on fruit diversity in the Solanaceae. J Exp Bot, 53, 2001-2022.

18. Stein, N., Prasad, M., Scholz, U., Thiel, T., Zhang, H., Wolf, M., Kota, R., Varshney, R.K., Perovic, D., Grosse, I. et al. (2007) A 1,000-loci transcript map of the barley genome: new anchoring points for integrative grass genomics. Theor Appl Genet, 114, 823-839. 


\section{FIGURES}

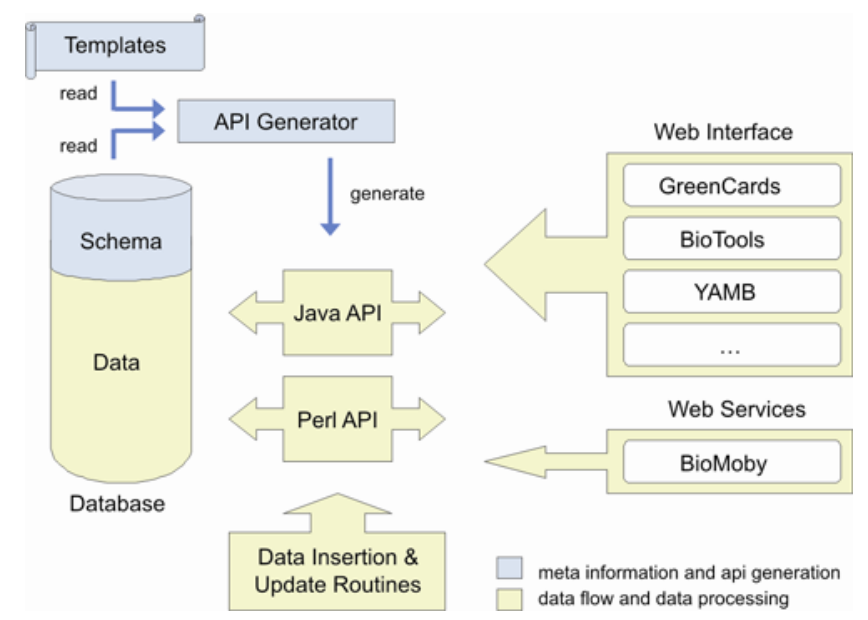

Figure 1. Schematic overview of the GabiPD application structure. The 'API generator' translates the 'Templates' using the database meta-information (shown in blue), generating the database application interface (Java and Perl API). The application logic (shown in yellow), i.e., Web Interface, WebServices and data manipulation routines, interacts with the 'Database' through the database application interface.

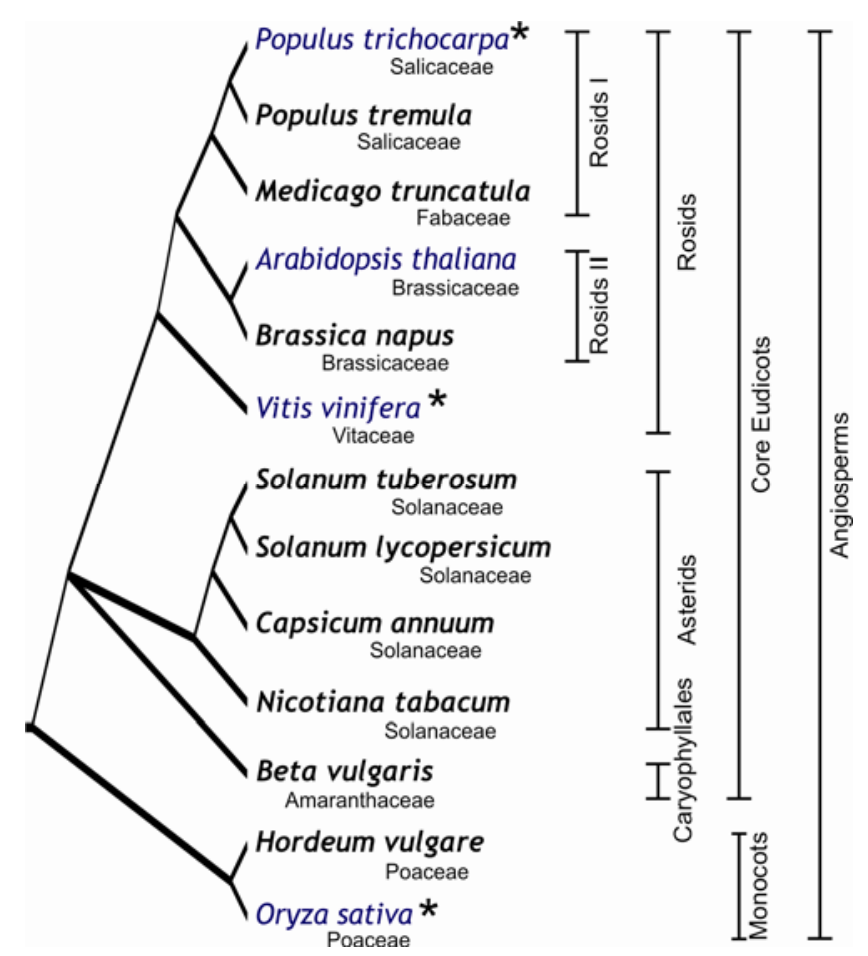

Figure 2. Phylogenetic tree depicting the evolutionary relationships among the species represented in GabiPD (15-17). Species for which whole-genome sequences and annotations are available are shown in blue. *Species that will soon be integrated in GabiPD. Species not shown: Solanum bulbocastanum, S. demissum, S. phureja, S. spegazzinii. 


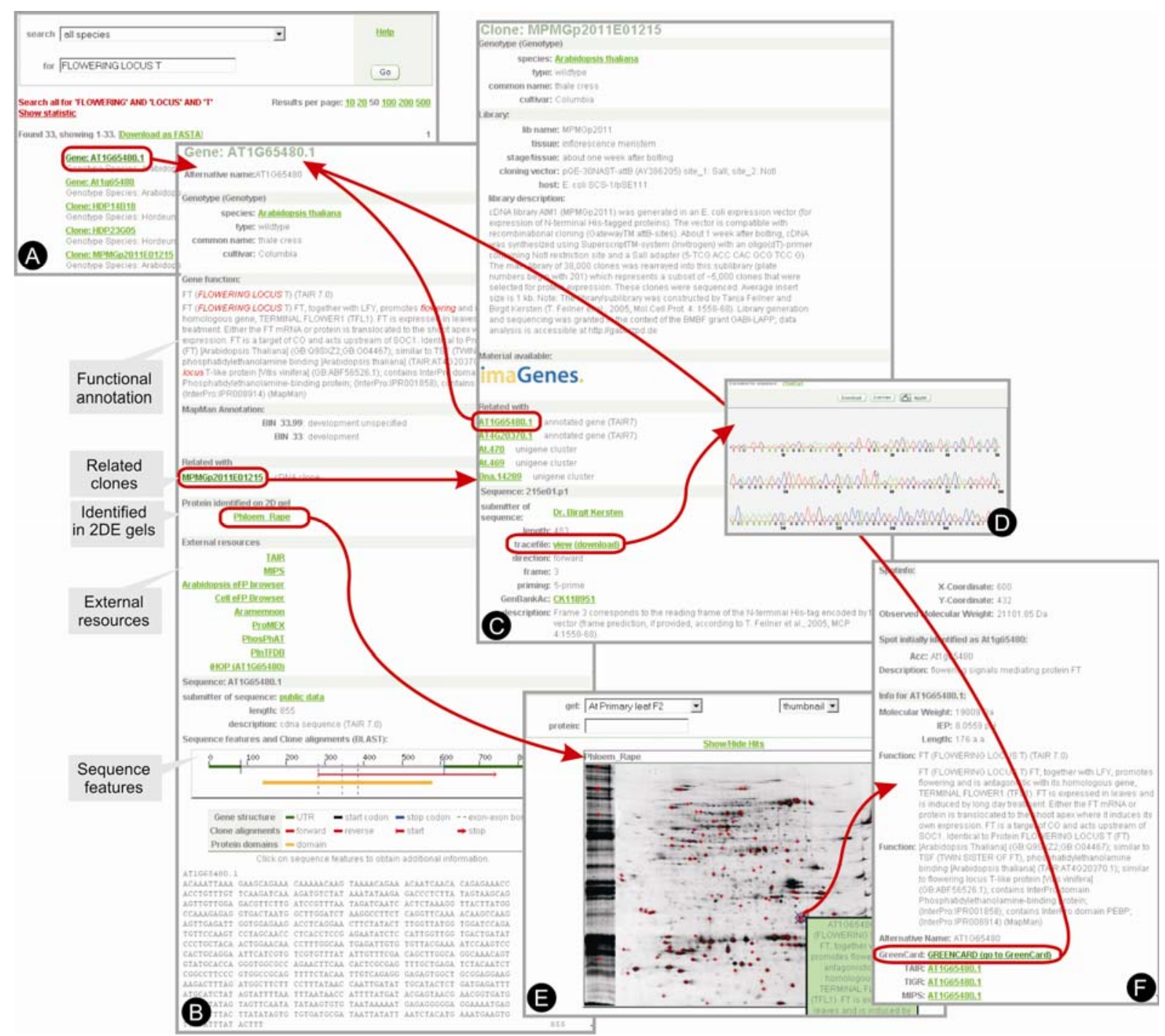

Figure 3. Example of a keyword search using GreenCards. (A) The user had performed a search for the keywords 'FLOWERING LOCUS T', which retrieves links to the GreenCards of Genes (genome annotation projects), Clones (ESTs) and Plants (mutant plant lines). (B) Display of the Gene GreenCard, corresponding to the Arabidopsis annotated gene AT1G65480.1. Here the users find high confidence matching EST sequences displayed in the 'Related with' section. Sequence features and the sequences themselves are displayed as well. The selected gene has a matching EST (Clone: MPMGp2011E01215), this Clone GreenCard is shown in (C) and links back to the Gene GreenCard and to the original EST trace file, displayed by JTrev (D). A protein spot in rapeseed (Brassica napus) has been identified by 2-DE/MS as the protein encoded by the retrieved gene, and a link directs the user to the 2DE-gel with the identified protein spot highlighted with blue cross-hair (E). The spot identified links to a description of the protein (F) that provides links to the original Gene GreenCard. 


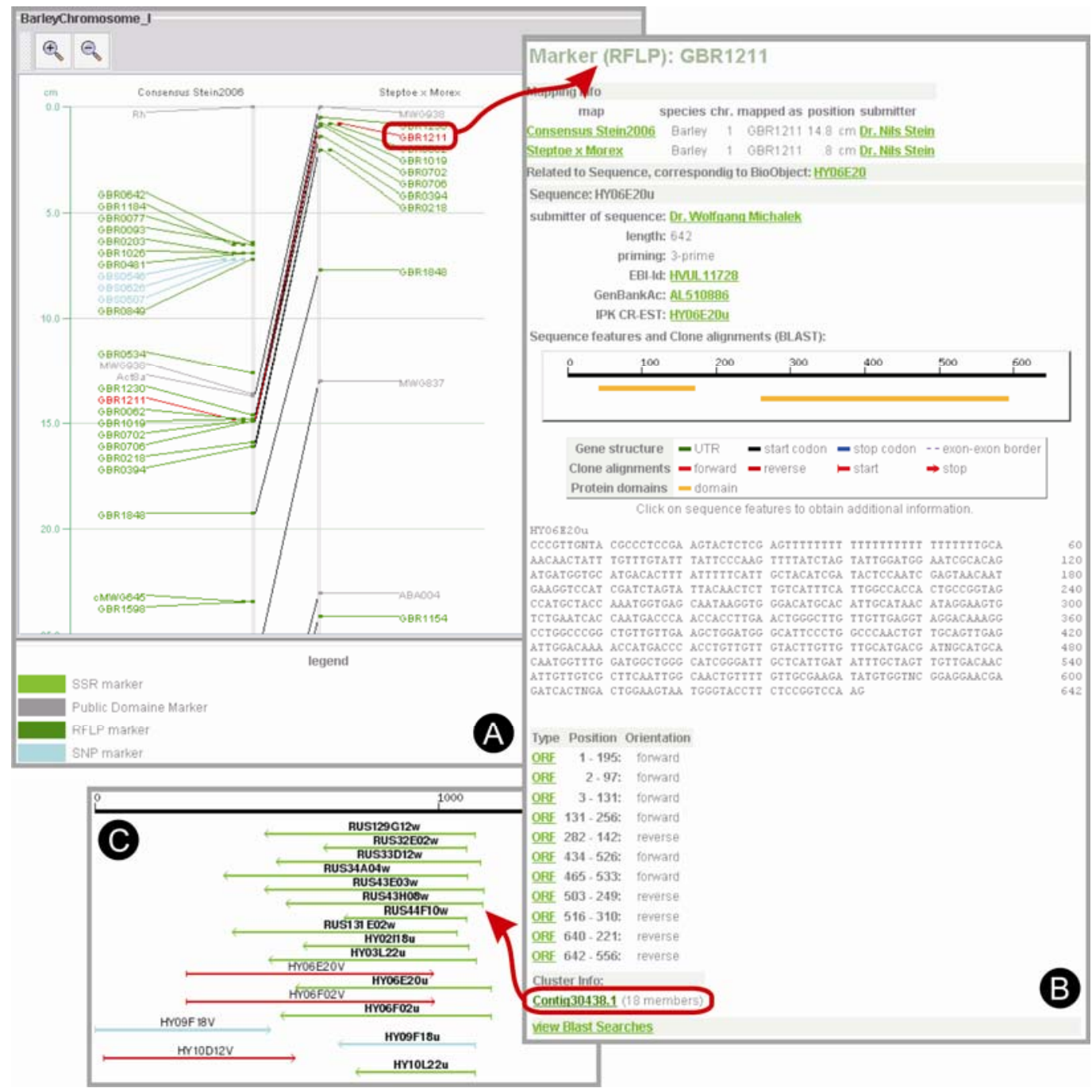

Figure 4. Visualisation of the genetic maps published by Stein et al. 2007 (18). (A) The region between 0cM and 25cM of barley chromosome I is shown; marker with single nucleotide polymorphisms (SNPs) are shown in light blue, marker with restriction fragment length polymorphisms (RFLPs) are shown in dark green. A selected marker is displayed in red, and links to the Marker GreenCard (B), which contains information on a related EST sequence therewith connecting genomic with transcriptomic information. With the EST description, cluster information is included (Contig30438.1) that links to the schematic representation of all ClusterContig members displayed onto the related consensus sequence (C). EST sequences that were selected from this ClusterContig as representatives for the new $27 \mathrm{~K}$ barley unigene set are shown in turquoise. 


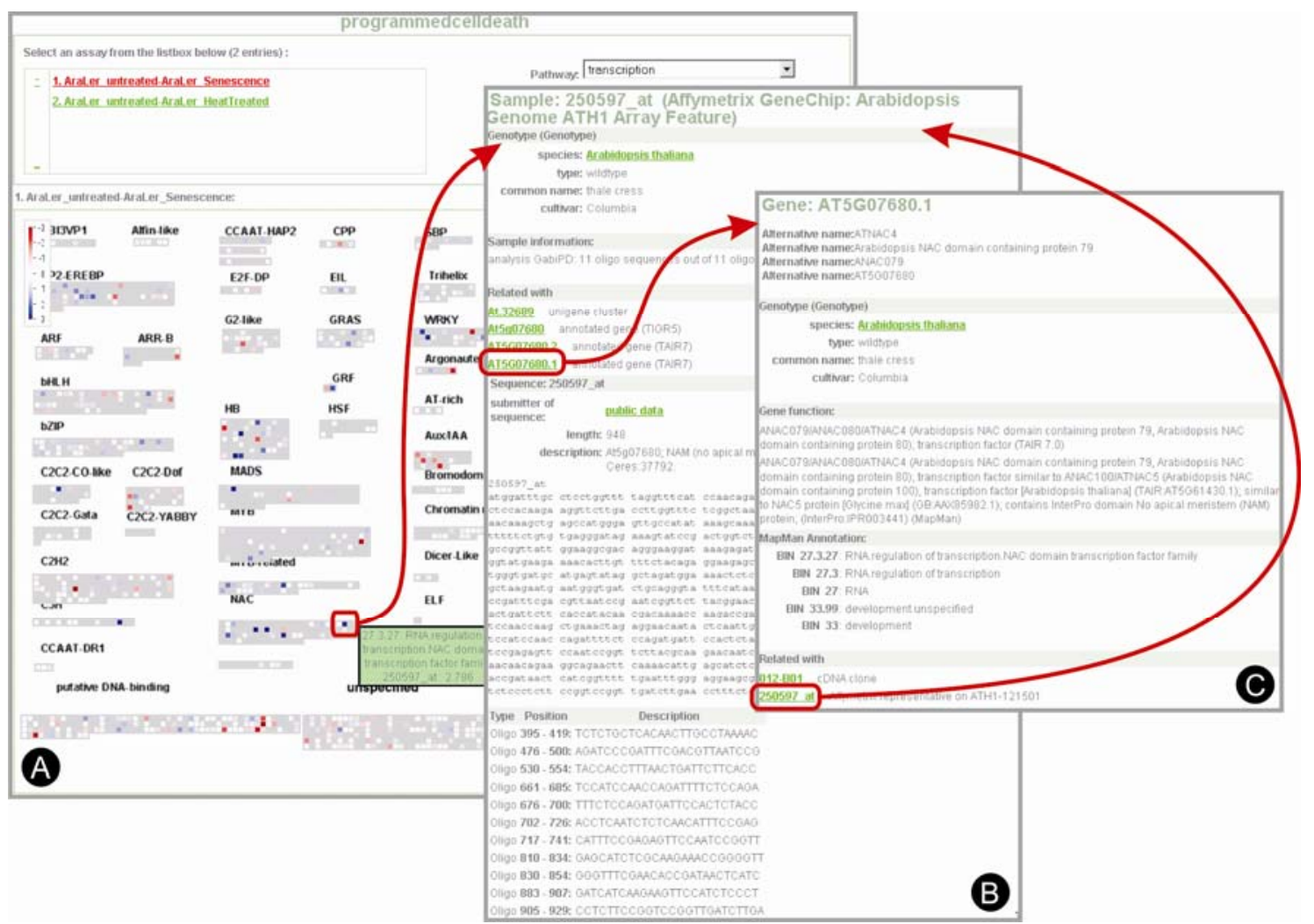

Figure 5. Visualisation of expression profile data in MapManWeb. (A) The Affymetrix ${ }^{\circledR}$ NASC Array experiment on programmed cell death in Arabidopsis is displayed (NASCArrays reference number: 30). MapManWeb allows the visualisation of expressed genes in different biological processes; here only probesets (i.e., genes) involved in transcription regulation are shown. (B) Details for a strongly down-regulated probeset, with links to the related Gene GreenCard in A. thaliana. (C) The Gene GreenCard for the selected gene (ANAC79) links back to the probeset of the Affymetrix ${ }^{\circledR}$ ATH1 array. 